\title{
Predicting the Induction Time of Hydrate Formation on a Water Droplet
}

\author{
M. Sarshar, F. Esmaeilzadeh and J. Fathikaljahi* \\ Chemical and Petroleum Engineering Department, School of Engineering, Shiraz University - Iran \\ e-mail: sarshar@shirazu.ac.ir - esmaeil@shirazu.ac.ir - fathi@shirazu.ac.ir
}

* Corresponding author

\begin{abstract}
Résumé - Prévision du temps d'induction de la formation d'hydrate sur la surface d'une goutte d'eau - Malgré l'importance du temps d'induction dans les études sur la formation d'hydrates, les experts n'en ont pas encore développé un modèle généralisé à cause de leur propriété stochastique. Dans cet article, le temps d'induction de la formation d'hydrate sur une goutte d'eau a été simulé pour un mélange de plusieurs gaz. Un modèle basé sur l'équation de la diffusion, où le taux de réaction est considéré par la théorie de nucléation, est proposé pour le transfert de masse à travers la surface de la goutte. La force motrice de la formation d'hydrate a été calculée à partir de la variation d'énergies libres de Gibbs du gaz, de l'eau et de l'hydrate. Le modèle a été validé par la comparaison des résultats de la modélisation avec les données expérimentales extraites de la littérature. Le temps d'induction de la formation d'hydrate a été calculé pour un mélange de $89,4 \%$ de méthane et 10,6 \% d'éthane sur une goutte d'eau d'un diamètre de $1,5-7 \mathrm{~mm}$ à $273,85 \mathrm{~K}$ et 5,1 MPa. On a trouvé que le temps d'induction est fortement sensible à la valeur de la force motrice. De plus, les temps d'induction ne dépendent pas de la taille des gouttes et sont inférieurs pour une goutte d'eau avec mémoire d'hydrate, comparativement à une goutte d'eau sans mémoire d'hydrate.
\end{abstract}

\begin{abstract}
Predicting the Induction Time of Hydrate Formation on a Water Droplet - Despite the importance of the induction time in hydrate formation studies, the scientists have not yet developed a generalized model due to its stochastic property. In this work, the induction time of hydrate formation of multicomponent gas mixtures on a water droplet has been modeled. Mass transfer through the droplet surface was modeled based on a diffusion equation where the rate of reaction was expressed based on the nucleation theory. Driving force for the hydrate formation was calculated based on the change in the Gibbs free energies of the gas, water and the hydrate. The model was validated by comparison of the predicted results with the experimental data from the literature. The induction time was calculated for hydrate formation from a mixture of $89.4 \%$ methane $+10.6 \%$ ethane on 1.5-7 mm in diameter of water droplets at 273.85 K and 3.6-5.1 MPa. It was found that, induction time is highly sensitive to the value of the driving force. Moreover, the induction times were not dependent on the size of the droplets and the induction time for the water droplets with hydrate memory was shorter than the droplets without hydrate memory.
\end{abstract}




\section{NOMENCLATURE}

\begin{tabular}{|c|c|}
\hline$a$ & Parameters of Equations 17,18 \\
\hline$A$ & Surface area $\left(\mathrm{m}^{2}\right)$ \\
\hline$A k$ & Kinetics parameter \\
\hline$A p$ & Particle surface $\left(\mathrm{m}^{2}\right)$ \\
\hline$b$ & Shape factor \\
\hline $\mathrm{Cl}$ & Langmuir constants \\
\hline$C$ & Concentration $\left(\mathrm{mol} / \mathrm{m}^{3}\right)$ \\
\hline$C p$ & Nucleation active center concentration $\left(\mathrm{m}^{-2}\right)$. \\
\hline$D$ & Diffusivity $\left(\mathrm{m}^{2} / \mathrm{s}\right)$ \\
\hline$f$ & Fugacity (bar) \\
\hline$g$ & Gibbs free energy $(\mathrm{J} / \mathrm{mol})$ \\
\hline$G$ & Growth rate constant \\
\hline$\Delta h$ & Enthalpy difference \\
\hline$J$ & Nucleation rate $\left(\mathrm{m}^{-3} \mathrm{~s}\right.$ or $\left.\mathrm{m}^{-2} \mathrm{~s}\right)$ \\
\hline$k$ & Boltzman constant \\
\hline$K$ & Mass transfer coefficient $(\mathrm{m} / \mathrm{s})$ \\
\hline$M$ & Molecular weight \\
\hline$N a$ & Avogadro number \\
\hline$n$ & Mole number \\
\hline$P$ & Pressure (bar) \\
\hline$r_{\mathrm{o}}$ & Cavity radial distance $\left({ }^{\circ} \mathrm{A}\right)$ \\
\hline$r$ & Drop radius \\
\hline$r c$ & Crystal radius (m) \\
\hline$R_{\mathrm{O}}$ & Cavity radius $\left({ }^{\circ} \mathrm{A}\right)$ \\
\hline$R$ & Gas Constant \\
\hline Rgi & Rate of gas consumption (mole $/ \mathrm{m}^{3} \mathrm{~s}$ ) \\
\hline$T$ & Temperature $(\mathrm{K})$ \\
\hline$V$ & Molar volume $\left(\mathrm{Cm}^{3} / \mathrm{mol}\right)$ \\
\hline$v c$ & Crystal volume $\left(\mathrm{m}^{3}\right)$ \\
\hline$x$ & Mole fraction \\
\hline$z_{\mathrm{o}}$ & Cavity coordination number \\
\hline$z$ & Gas mole fraction in hydrate \\
\hline
\end{tabular}

\section{Greek Letters}

$\delta \quad$ Parameters of equation 7

$\varepsilon_{\mathrm{o}} \quad$ Kihara parameter of equation 7

$\varepsilon \quad$ Kinetics parameter

$\varepsilon^{1} \quad$ Leonard Jones parameter

$\gamma \quad$ Activity coefficient

$\mu \quad$ Chemical potential $(\mathrm{J} / \mathrm{mol})$

$\theta \quad$ Cavity occupation fraction

$\sigma_{\mathrm{o}} \quad$ Kihara parameter of equation 7

$\sigma \quad$ Surface energy $\left(\mathrm{J} / \mathrm{m}^{2}\right)$

$\sigma^{1} \quad$ Leonard Jones parameter

$v \quad$ Cavity number per water molecules

$\psi \quad$ Shape factor

$\omega \quad$ Partition function used in equation 6

\section{Subscripts}

$\begin{array}{ll}E & \text { Equilibrium } \\ H & \text { Hydrate } \\ I & \text { Counter } \\ j & \text { Counter } \\ k & \text { Counter } \\ w & \text { Water }\end{array}$

\section{Superscripts}

$\begin{array}{ll}\text { aq } & \text { Aqueous phase } \\ \text { g } & \text { Gas phase } \\ \text { h } & \text { Hydrate phase } \\ \text { MT } & \text { Empty hydrate lattice } \\ 0 & \text { Standard state }\end{array}$

\section{INTRODUCTION}

Gas hydrates crystals are non-stoichiometric compounds and consist of polyhedral water cavities that are stabilized by the presence of gaseous components. Three types of structures are known as structure I, II and H (Sloan, [1]). A serious problem in the oil and gas industries is the plugging of natural gas transportation lines because of the solid nature of hydrates. Natural gas hydrate occurrence is a significant factor to be considered during oil and gas exploration, production and transportation (Englezos, [2]; Sloan, [1]).

In natural gas production, both gas and aqueous phases are usually present. In cold areas and subsea pipelines, in the temperature range of $5-25^{\circ} \mathrm{C}$, gas hydrates may form, which is undesirable due to the possibility of plugging of flow lines. There are various options to prevent hydrate crystallization. These options include heating, insulation, water removal, and the use of thermodynamic inhibitors. In recent years, the use of kinetic inhibitors (surfactants or polymers) has become an attractive option. The basic premise of a kinetic inhibitor is to influence the induction time in gas hydrate crystallization, the growth rate of the separate hydrate crystallites or the agglomeration of these crystallites. The concentration of the kinetic inhibitors in the aqueous phase is relatively low (less than $1 \mathrm{wt} \%)$. In thermodynamic inhibition, the gas-water-hydrate equilibrium curve is shifted; the shift requires a substantial amount of thermodynamic inhibitor (often more than $20 \mathrm{wt} \%)$.

Kvamme [3] proposed a new hydrate formation theory based on the hypothesis that initial hydrate formation takes place at the gas-liquid interface towards the gas side. The theory was modeled and compared to experimental data. Kvamme [4] published a revised version of the model where evaluation of cluster distribution and cluster stability analysis were included. 
Kashchiev and Firoozabadi [5] analyzed the kinetics of the initial stage of crystallization of one-component gas hydrates in aqueous solutions. The temporal evolution of the volume of hydrate crystallized and the moles of gas consumed were determined. Expressions were derived for the supersaturation dependence of the hydrate crystallite growth rate and the induction time in hydrate crystallization. These expressions were used for revealing how additives in the solution that act as kinetic inhibitors of hydrate crystallization can affect the induction time of the process. The obtained results were applied to crystallization of methane, ethane and cyclopropane hydrates.

Lee et al. [6] studied gas hydrate formation and decomposition on water droplets using a $89.4 \%$ methane- $10.6 \%$ ethane mixture, and a $90.1 \%$ methane- $9.9 \%$ propane mixture. The experiments were carried out in a new apparatus suitable for morphology studies. In each experiment, they measured simultaneously the induction time of hydrate formation of two or three different size drops in the apparatus. Hydrate formation was studied in the first stage with fresh water and in the other stages with hydrate experienced droplets. After the first stage of hydrate formation, pressure was reduced to the value less than the equilibrium pressure to decompose hydrate completely. After $1 \mathrm{~h}$ of decomposition, pressure was increased again to the formation pressure to reform the hydrate. They found that the induction time was much shorter when the water had hydrate memory. Further, the droplet size had no noticeable effect on the induction time and on the macroscopic crystal growth morphology for hydrates from the methane-ethane mixture. Moreover, the smaller droplets during hydrate reformation showed an extensive hydrate growth and looked like snow-flakes. A brief description of Lee et al.'s [6] experiments using a $89.4 \%$ methane-10.6\% ethane mixture is given in Table 1 .

\section{TABLE 1}

The results of Lee et al.'s experiments using $89.4 \%$ methane-10.6\% ethane mixture at $273.85 \mathrm{~K}$

\begin{tabular}{c|c|c|c|c}
\hline Experiment & \multirow{2}{*}{$\begin{array}{c}\text { Formation } \\
\text { pressure }\end{array}$} & \multirow{2}{*}{$\begin{array}{c}\text { Droplet size } \\
(\mathrm{mm})\end{array}$} & \multicolumn{2}{|c}{ Induction time (min) } \\
\cline { 4 - 5 } & $(\mathrm{MPa})$ & & Fresh water & Hydrate memory \\
\hline 1 & 5.1 & $1.5,2.5,6$ & $47-48$ & $0.5-1.8$ \\
\hline 2 & 5.1 & $5^{*}$ & $35.7-35.8$ & $2.2-2.5$ \\
\hline 3 & 5.1 & $1.5,2.5,6$ & $94-95$ & $0.5-1.8$ \\
\hline 4 & 3.6 & 5,7 & 79.3 & $2.0-2.3$ \\
\hline
\end{tabular}

* Two different shapes of droplets.

They observed by sequential pictures, generated by timelapse videos, the time required for hydrate to cover the water droplet surface ranged from 10 to $23 \mathrm{~s}$ and was shorter when there was gas-phase agitation (mixing). They postulated that the growth is occurred in two stages [6].
Rodger [7] studied the memory effects of methane hydrate formation and showed using molecular dynamic simulation that enhanced levels of ice and clathrate structures were formed in the liquid water when the hydrate decomposes.

Thompson et al. [8] studied by Neutron diffraction the water structure at the early, medium, and late periods of methane clathrate hydrate formation and decomposition. They used empirical potential structure refinement computer simulations to analyze the neutron diffraction data and extracted from the data the water structure in the bulk methane hydrate solution. Their results, highlighted the significant changes in the water structure of the remaining liquid at various stages of hydrate formation and decomposition, and gave further insight into the way in which hydrates form. The results also had important implications on the memory effect, suggesting that the water structure in the presence of hydrate crystallites was significantly different at equivalent stages of forming compared to decomposing. For these systems, there was no detectable change in the water structure or the methane hydration shell before hydrate formation and after decomposition. Based on the results presented in their paper, it is clear that the local water structure is affected by the presence of hydrate crystallites, which may in turn be responsible for the "history" or "memory" effect. Hence, they explained memory effect by a low concentration of hydrate crystallites remaining in solution after the hydrate appears to have melted. This leads to a locally modified water structure, which in turn allows the hydrate to reform more quickly [8].

In this work, the induction time of hydrate formation of multicomponent gas mixtures on a water droplet has been modeled. Mass transfer through the water surface was modeled based on a diffusion equation where the rate of reaction was expressed based on the nucleation theory. Driving force of the hydrate formation reaction was calculated based on the work of Kashchiev and Firoozabadi [5]. The induction time, which is the time needed for the formation of a detectable portion of hydrate, was calculated for the mixture of methane and ethane at $273.85 \mathrm{~K}$ and 3.6-5.1 MPa. It was shown that the induction time is highly sensitive to the value of the driving force. The model is validated by comparison of the calculated results with the experimental data through the literature (Lee et al. [6]).

\section{MATHEMATICAL MODELING OF HYDRATE FORMATION}

Hydrate is formed on a water droplet in the presence of a gas mixture at the system temperature and pressure higher than the equilibrium formation pressure. When gas is brought into contact with a water droplet, the gas components diffuse toward the water-gas interface, absorbed at the interface and diffuse to the center of the drop. At the interface due to the higher value of supersaturation, an unstable hydrate complex 


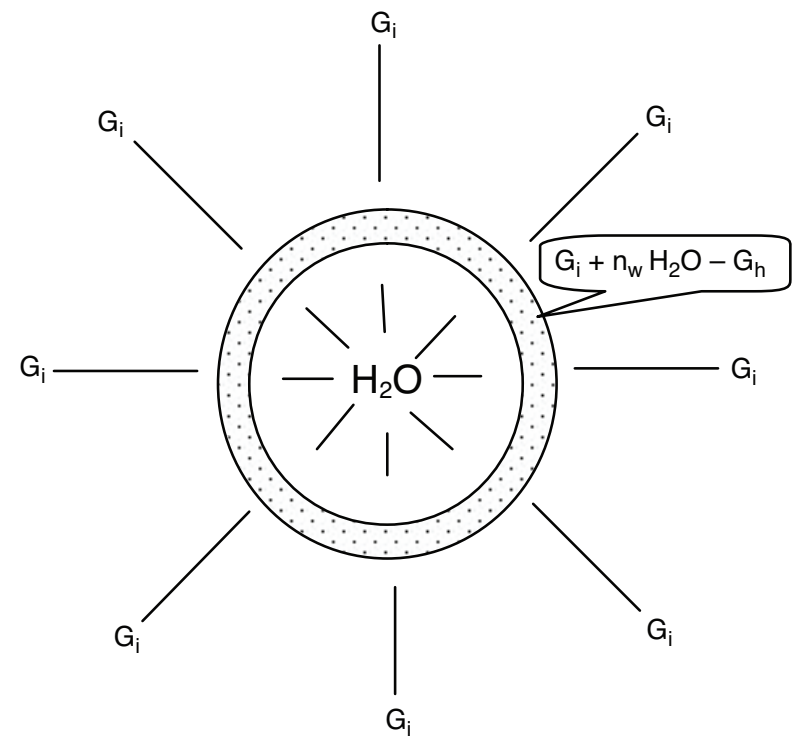

Figure 1

Schematic representation of hydrate formation on a water droplet.

is formed. Then, the hydrate complex is separated from the aqueous phase and forms hydrate nucleus. Finally, by the attachment of the separate nucleus a detectable portion of hydrate is formed. Figure 1 represents the mechanism of hydrate formation on a water droplet. In this figure, the thickness of hydrate reacting zone is drawn large enough to distinguish it from the surface of the drop. Hydrate has not yet been formed.

Based on the mechanism shown in Figure 1, a model is developed for the gas concentration inside the water droplet. The model is a second order diffusion equation with the following initial and boundary conditions.

$\partial C_{i}^{a q} / \partial t=D_{i w}^{a q}\left(\partial / r^{2} \partial r\left(r^{2} \partial C_{i}^{a q} / \partial r\right)\right) \quad i=1, \ldots, n$

Initial condition: $\quad t=0$ and $0 \leq r \leq r_{\text {drop }} \quad C_{i}^{a q}=0$

Boundary conditions: $t=0$ and $r=0 \quad \partial C_{i}^{a q} / \partial r=0$

Boundary conditions:

$t=0$ and $r=r_{\text {drop }}-D_{i w}^{a q} \partial C_{i}^{a q} / \partial r=K m_{i}^{g}\left(C_{i}^{g}-C e_{i}^{g}\right)-R g_{i}^{h}$

In the above equations, $C_{i}^{a q}$ and $D_{i w}{ }^{a q}$ are concentration and diffusivity of the gas component $i$ in the aqueous phase. $C_{i}{ }^{g}$ and $\mathrm{Km}_{i}{ }^{g}$ are concentration and mass transfer coefficient of component $i$ in the gas. $C e_{i}{ }^{g}$ and $R g_{i}{ }^{h}$ are the interfacial concentration and the rate of gas consumption at experimental temperature and pressure.

\subsection{Rate of Gas Consumption in Hydrate Formation Reaction}

The rate of gas consumption of hydrate formation reaction at the liquid-gas interface is calculated based in the following equation [9].

$$
R g_{i}^{h}=d n_{i}(t) / d t=V_{C}(t) J(t) z(t)_{i}^{h} / N_{a} v_{h}
$$

In the above equation, $R g_{i}^{h}\left(\mathrm{~m}^{3} / \mathrm{m}^{2} . \mathrm{s}\right), z$ and $J\left(\mathrm{~m}^{-2} \cdot \mathrm{s}^{-1}\right)$ are gas consumption, gas composition and nucleation rates, respectively. $V_{c}\left(\mathrm{~m}^{3}\right)$ is the individual hydrate crystal volume and is calculated by the following expression [9].

$$
V_{C}(t)=b r_{c}^{3}(t)
$$

$b$ is the dimensionless shape factor and is defined by $b=4 \pi / 3$ for spherical crystal, $b=8$ for cubes, $b=4 \pi / 3 \psi(\theta)$ for caps on a solid substrate with wetting angle $\theta(0 \leq \theta \leq 180)$. Wetting angle is used to identify wetting property of the nucleus on a solid substrate [12]. Higher wetting tendency is characterized by lower wetting angle.

$b=\left[\psi(\theta)+\psi\left(\theta_{0}\right)\left(\sin \theta / \sin \theta_{0}\right)^{3}\right]$ for lenses at the solution/ gas interface with $\theta, \theta_{0}$ characterizing hydrate/solution and hydrate/gas contacts angles, respectively. The function $\psi(x)$ is defined by equation 4 [10]. Figure 2 shows schematically nucleus formation at the liquid gas interface and on a solid substrate.

$$
\psi(x)=(1 / 4)(2+\cos x)(1-\cos x)^{2}
$$

$\theta$ and $\theta_{0}$ are calculated by the following equations when hydrate nucleus is formed at the liquid gas interface [12].

$$
\begin{gathered}
\cos \theta=\left(\sigma_{S g}^{2}+\sigma^{2}-\sigma_{h g}^{2}\right) / 2 \sigma_{S g} \sigma \\
\cos \theta_{0}=\left(\sigma_{S g}^{2}-\sigma^{2}+\sigma_{h g}^{2}\right) / 2 \sigma_{S g} \sigma_{h g}
\end{gathered}
$$

where $\sigma_{s g}$ and $\sigma_{h g}$ are the specific surface energies of the liq$\mathrm{uid} / \mathrm{gas}$ and hydrate/gas interfaces, respectively. $\sigma$ is the specific surface energy of the hydrate/solution interface [5]. The numerical values of sigmas are shown in Table 2.

$r_{c}(t)$ is the hydrate dimension and is defined by Equation 7 based on the mechanism of power law growth rate.

$$
r_{c}(t)=(G t)^{m}
$$

in which $m>0$ is a number and $G\left(\mathrm{~m}^{1 / m} / \mathrm{s}\right)$ is the growth constant and is calculated based on the kinetics considerations.

$$
G\left(\mathrm{~m}^{2} / \mathrm{s}\right)=2 \varepsilon v_{h} D_{i w}^{a q} C_{e}\left(e^{\Delta g / k T}-1\right) \quad \mathrm{m}=1 / 2
$$

Equation 8 is used for the growth by undisturbed volume diffusion of dissolved gas toward a spherical crystallite (Volmer [10], Mullin [11] and Kashchiev and Firrozabadi [5]).

In the above equations, $C_{e}$ is equilibrium solubility of the gas component and $D_{i w}^{a q}$ is the gas diffusivity in the aqueous solution, respectively. $J(t)=J$, the nucleation rate is given by 


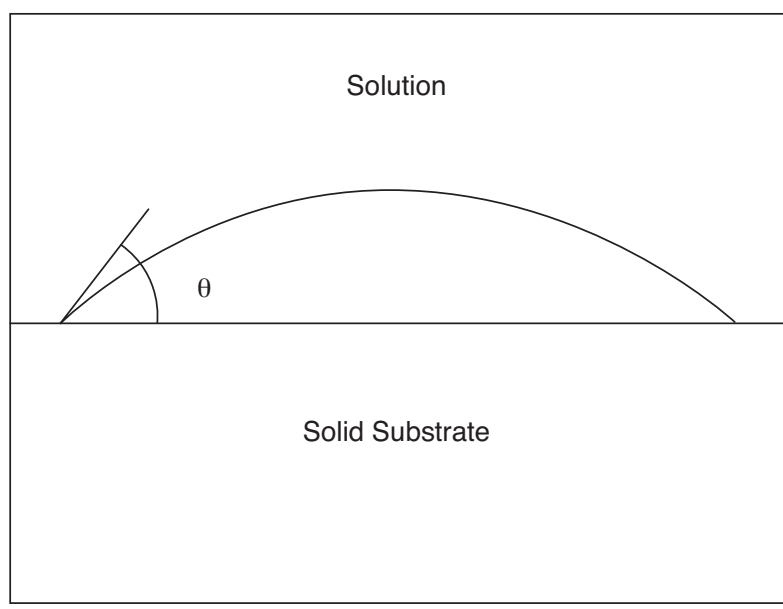

a)

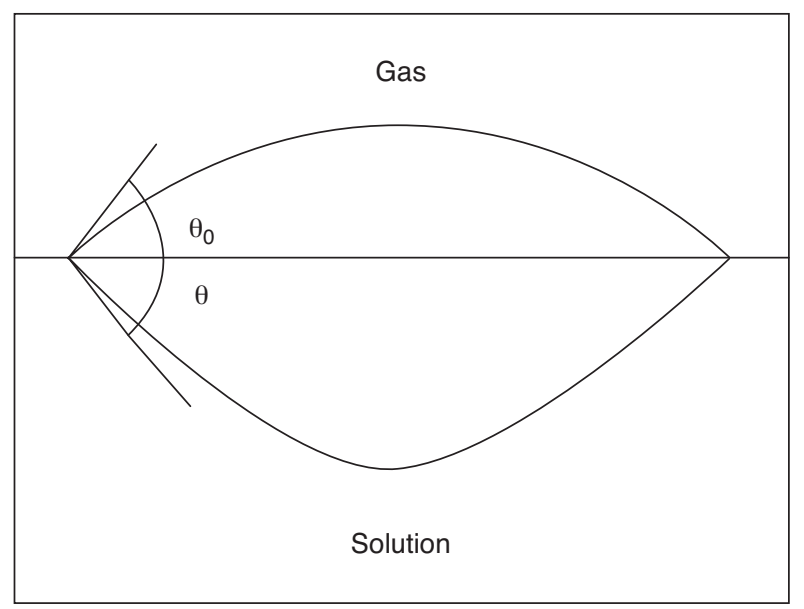

b)

Figure 2

Schematic representation of nucleus formation on a) solid substrate and b) at the liquid gas interface.

Kashchiev and Firoozabadi [12] and is defined by Equation 9. The work for cluster formation is augmented in this equation.

$$
J=A k e^{\Delta g / k T} \exp \left(-4 c^{3} v_{h}^{2} \sigma_{e f}^{3} / 27 k T \Delta g^{2}\right)
$$

Here, $c$ is numerical shape factor and is equal to $(36 \pi)^{1 / 3}$ and $\sigma_{e f}\left(\mathrm{~J} / \mathrm{m}^{2}\right)$ is an effective specific surface energy which is expressed by the following equation:

$$
\sigma_{e f}=\Psi \sigma
$$

$\Psi$ is a number between 0 and 1 and is equal to $\Psi=(\psi(\theta))^{1 / 3}$ for cap-shaped hydrae nucleus on a solid substrate and is equal to $\Psi=\left[\psi(\theta)+\psi\left(\theta_{0}\right)\left(\sin \theta / \sin \theta_{0}\right)^{3}\right]^{1 / 3}$ for lens shaped hydrate nucleus at the solution/gas interface [12].

$A k$ is a kinetic parameter and is defined based on the mechanism of attachment of hydrate building units to the nucleus and mechanism of nucleation (Kashchiev and Firoozabadi [12]). For heterogeneous nucleation on solid substrate or at the liquid/gas interface without nucleation active center, the $A k$ parameter is calculated by Equation 11 and with nucleation active center, the Ak parameter is calculated by Equation 12 [12].

$$
\begin{gathered}
A k=z \varepsilon(4 \pi c)^{1 / 2} v_{h}^{1 / 3} D^{a q} C_{e} \dot{n}^{1 / 3} / a_{W} \\
A k=z \varepsilon(4 \pi c)^{1 / 2} v_{h}^{1 / 3} D^{a q} C_{e} \dot{n}^{1 / 3} C_{P}
\end{gathered}
$$

In the above equations, $z=0.01-1$ is the Zeldovich factor, $\varepsilon=1$ and $a_{w}$ is the surface area of a water molecule. $C_{p}$ is concentration of the nucleation active center at the liquid gas interface. $\dot{n}$ is the number of building units constituting a nucleus and is calculated by the following expression [9].

$$
\dot{n}=8 c^{3} v_{h}^{2} \sigma_{e f}^{3} / 27 \Delta g^{3}
$$

$\Delta g$ is the supersaturation or the driving force of hydrate formation which is discussed in the next section.

\subsection{Driving Force of Hydrate Formation}

According to the work of Anklam and Firrozabadi [13], hydrate formation reaction is considered as an aqueous phase reaction where a unit cell of hydrate is formed from different gas molecules by the following reaction:

$$
z_{1} G_{1}+z_{2} G_{2}+\ldots+z_{w} \mathrm{H}_{2} \mathrm{O} \rightarrow z_{1} G_{1}, z_{2} G_{2}, \ldots, n_{w} \mathrm{H}_{2} \mathrm{O}
$$

where $z_{i}$ is the composition of gas molecules in the hydrate phase, $G_{i}$ is the gas molecules and $n_{w}$ is the hydration number. The driving force expression for the above reaction is given by:

$$
\begin{aligned}
& \Delta g=\sum_{i=1} z_{i}\left(T, P_{e}, z_{e}\right) k T \ln \left[\hat{f} g_{i}(T, P, y) / \hat{f}_{i}\left(T, P_{e}, y\right)\right] \\
& +n_{w} v_{w}\left(P-P_{e}\right)-v_{h}\left(P-P_{e}\right)
\end{aligned}
$$

In deriving the above equation, it is assumed that hydrate composition is independent of the supersaturation and it is similar to the equilibrium composition. In the above equation, $T, P_{e}$ and $P$ are the system temperature, equilibrium hydrate pressure and the system pressure, respectively. $f g_{i}, y_{i}$ and $z_{i}$ are gas fugacity, gas composition and the hydrate equilibrium composition, respectively. 


\subsection{Equilibrium Hydrate Formation}

Van der Waals and Platteeuw [14] developed a model for the calculation of gas occupancy in the hydrate cavities and a model for water chemical potential in hydrate phase based on statistical thermodynamics. Further, Parrish and Prausnitz [15] extended Van der Waals and Platteeuw [14] models for hydrate equilibrium calculations of multicomponent gas mixture. They derived Equations 16 and 17 for calculating the chemical potential of water in hydrate based on statistical thermodynamics.

$$
\begin{gathered}
\mu_{w}^{H} / R T=\mu_{w}^{M T} / R T+\sum_{i} v_{i} \operatorname{Ln}\left(1-\sum_{k} \theta_{k i}\right) \\
\theta_{k i}=C l_{k i} \hat{f}_{k} /\left(1+\sum_{j} C_{j i} \hat{f}_{j}\right)
\end{gathered}
$$

In the above equations, $C l_{k i}$ is the langmuir constant for component $k$ in cavity $i, v_{i}$ is number of cavity $i$ per water molecules in a hydrate structure and $\theta_{k i}$ is fractional occupation of cavity $i$ by component $k$. Superscript H and MT are used for hydrate and empty hydrate crystal, respectively. Subscript $k$ and $j$ are used for component number and $i$ is used for cavity type. $\theta_{k i}$ or fractional occupation of cavity $i$ by component $k$ is calculated by Equation 17 .

Langmuir constants are calculated based on the Kihara core potential with Lennard-Jones De Vonshire theory by Equations 18-20.

$$
C l_{k i}=4 \pi / k T \int_{0}^{R o-a_{i}} \exp \left(-\omega\left(r_{o}\right) / k T\right) r^{2} d r
$$

$\omega\left(r_{o}\right)=2 z_{o} \varepsilon_{o k}$

$\left[\sigma_{k}^{12} / R_{o}{ }^{11} r_{o}\left(\delta^{10}+\delta^{11} a_{k} / R_{o}\right)-\sigma_{k}^{6} / R_{o}^{5} r_{o}\left(\delta^{4}+\delta^{5} a_{k} / R_{o}\right)\right]$

$\delta^{N}=1 / N\left[\left(1-r_{o} / R_{o}-a_{k} / R_{o}\right)^{-N}-\left(1+r_{o} / R_{o}-a_{k} / R_{o}\right)^{-N}\right]$

$a_{k}, \varepsilon_{0 k}$ and $\sigma_{0 k}$ are the Kihara parameters, $R_{o}$ is cavity radius and $z_{0}$ is coordination number. The values of these parameters are presented by Sloan [1]. Chemical potential of water in the aqueous phase is calculated by Equation 21.

$$
\begin{aligned}
& \left(\mu_{w}^{M T}-\mu_{w}^{a q}\right) / R T=\Delta \mu_{w}^{0} / R T_{0}-\int_{T 0}^{T}\left(\Delta h_{w} / R T^{2}\right) d T \\
& +\int_{0}^{p}\left(\Delta v_{w} / R T\right) d P-\operatorname{Ln}\left(\gamma_{w} x_{w}\right)
\end{aligned}
$$

$\Delta u_{w}^{0}, \Delta h_{w}$ and $\Delta v_{w}$ are difference between the standard chemical potential, enthalpy and molar volume of water in the aqueous phase and empty hydrate crystal. The values of these parameters are presented by Sloan [1]. $\gamma_{w}$ and $x_{w}$ are the activity coefficient and mole fraction of water, respectively. $\mu_{w}^{M T}$ is a reference value and represents the chemical potential of water in an empty hydrate crystal.
Gas fugacities are calculated by Soave-Redlich-Kowong (SRK) equation of states (Soave [16]). Activity coefficients of the dissolved gases are set equal to unity because of the low solubility of gases in the aqueous phase.

The equilibrium condition for the hydrate formation is described as the equality of the water chemical potentials in the hydrate and in the aqueous phases.

$$
\mu_{w}^{h}=\mu_{w}^{a q}
$$

In the other words, the water chemical potential in the hydrate phase and in the aqueous phases must be the same at equilibrium temperature and pressure. Water chemical potentials of the hydrate phase and the aqueous phases are calculated by Equations 16 and 21, respectively.

\subsection{Transport Parameters}

Liquid phase diffusivities are calculated by Wilk-Chang equations [17] where, $D_{i w}^{a q}, M_{w}$ and $\eta_{w}$ are aqueous phase diffusivity $\left(\mathrm{m}^{2} / \mathrm{S}\right)$, water molecular weight $(\mathrm{g} / \mathrm{gmol})$ and water viscosity (cp), respectively. $T$ is the absolute temperature $(\mathrm{K})$ and $v_{i}$ is the solute molar volume at its normal boiling point $\left(\mathrm{cm}^{3} / \mathrm{mol}\right)$. $\phi$ is a fixed parameter and is equal to 2.6 [17].

$$
D_{i w}^{a q}=7.4 \times 10^{-12}\left(\phi M_{w}\right)^{1 / 2} T / \eta_{w} v_{i}^{0.6}
$$

In order to express the mass transfer coefficient of the agitated gas phase, one have to assign a large value to $S h$ number. So any number that eliminates the mass transfer resistance in the gas phase is good enough for the calculations. Therefore we find $S h$ number equals to 10 in our calculations where $S h$ is a dimensionless number and is defined by $S h=K_{i}^{g} d / D_{i j}{ }^{g}$. In this equation, $d$ is the drop diameter and $D_{i j}{ }^{g}$ is the mutual diffusion coefficient of the gaseous components and is calculated by the following expression [17]

$$
D_{i j}^{g}=2.66 \times 10^{-7} T^{3 / 2} / P M_{i j}^{0.5} \sigma_{i j}^{l 2} \Omega_{D}
$$

In this equation, $\Omega$ is an energy parameter, $T$ and $P$ are the absolute temperature (K) and pressure (bar). $M_{i j}$ and $\sigma_{i j}$ are the average values of molecular weight and molecular diameter and calculated by the following equations. $\varepsilon$ and $\sigma$ are Leonard Jones parameters and are presented by Bird et al. [17].

$$
\begin{gathered}
M_{i j}=2\left[1 / M_{i}+1 / M_{J}\right]^{-1} \\
\sigma_{i j}^{l}=\left(\sigma_{i}^{l}+\sigma_{j}^{l}\right) / 2 \\
\varepsilon_{i j}^{l}=\sqrt{\varepsilon_{i}^{l} \varepsilon_{j}^{l}} \\
\Omega_{D}=\frac{1.06036}{t^{\bullet}{ }^{0.15610}}+\frac{0.193}{\exp \left(0.47635 t^{\bullet}\right)} \\
+\frac{0.47635}{\exp \left(1.52996 t^{\bullet}\right)}+\frac{1.76474}{\exp \left(3.8941 t^{\bullet}\right)} \\
t^{\bullet}=T / \varepsilon_{i j}^{l}
\end{gathered}
$$




\subsection{Solution Method of the Mathematical Models}

Concentration profiles of the gas components inside the water droplet are determined using backward difference time domain method to solve the diffusion Equation 1. The resulting equation and its initial and boundary conditions are defined by Equation 30. $i, j$ stand for time and length steps, respectively.

$C_{i+1, j+1}^{k}(2 / j+1)+C_{i+1, j}^{k}(1 / \alpha+2 / j+2)-C_{i+1, j-1}^{k}=C_{i, j}^{k} / \alpha$,

$\Delta=\mathrm{D} \Delta t / \Delta r^{2}$

$C_{0, j}=0 \quad$ for all $J$

for all $i$

Initial condition

$C_{i, 0}=C_{i, 1}$ Boundary conditions

$C_{i+1, n}^{k}-C_{i+1, n-1}^{k}=-(\Delta r / D)\left(K^{g}\left(C_{i+1, g}^{k}-C e_{i+1, g^{*}}^{k}\right)+R g_{i+1}^{h}\right)$ for all $i$.

The rate of gas consumption at each time step is calculated by Equation 2, where $N_{a}$ and $v_{h}$ stand for Avogadro number and hydrate volume, respectively. The numerical values of $v_{h}$ and $N_{a}$ are shown in Table 2.

Induction time is defined by the time required for the conversion of $1 \%$ of water to hydrate based on the polynuclear mechanism [5]. The converted water to hydrate is calculated by integrating the hydrate formation rate with respect to time multiplied by the hydration number.

TABLE 2

Numerical values of the major parameters

\begin{tabular}{l|c|c}
\hline Parameter & Value & Ref. \\
\hline$D_{i \text {-Water }}\left(\mathrm{m}^{2} / \mathrm{s}\right)$ & $8.9 \times 10^{-10}$ & Eq. 24 \\
\hline$D_{i j \text {-Gas }}\left(\mathrm{m}^{2} / \mathrm{s}\right)$ & $2.0 \times 10^{-7}$ & Eq. 25 \\
\hline $\mathrm{K}_{\mathrm{CH}_{4}-\mathrm{C}_{2} \mathrm{H}_{6}}(\mathrm{~m} / \mathrm{s})$ & $3.54 \times 10^{-4}$ & $S h=10$ \\
\hline$N_{a}\left(\mathrm{~mol}^{-1}\right)$ & $6.02 \times 10^{23}$ & {$[5]$} \\
\hline$v_{h}(\mathrm{SI})\left(\mathrm{nm}^{3}\right)$ & 0.216 & {$[5]$} \\
\hline$\sigma\left(\mathrm{J} / \mathrm{M}^{2}\right)$ & $18.5 \times 10^{-3}$ & {$[5]$} \\
\hline$\sigma_{h g}\left(\mathrm{~J} / \mathrm{M}^{2}\right)$ & $80 \times 10^{-3}$ & {$[5]$} \\
\hline$\sigma_{s g}\left(\mathrm{~J} / \mathrm{M}^{2}\right)$ & $70 \times 10^{-3}$ & {$[5]$} \\
\hline$P_{e}(\mathrm{MPa})$ & 1.58 & Sect. 1-3 \\
\hline
\end{tabular}

Driving force $(\Delta g)$ of hydrate formation reaction is calculated by Equation 15 at experimental conditions of $273.85 \mathrm{~K}$ and 3.6-5.1 MPa. Driving force is used in calculating the growth rate constant and the nucleation rate.

The equilibrium hydrate formation pressure shown in Table 2 is calculated based on the method described in Section 2.3 for a $89.4 \% \mathrm{CH}_{4}+10.6 \% \mathrm{C}_{2} \mathrm{H}_{6}$ gas mixture at $273.85 \mathrm{~K}$.

\section{RESULTS AND DISCUSSION}

Hydrate clusters are created initially and then by the attachment, a stable nucleus is formed. The time required for the conversion of $1 \%$ of water to hydrate is equal to induction time [5]. Induction time is calculated for the gas mixture with both fresh water and water formed from melted hydrate. In both cases, the effect of the influencing parameters on the induction time is examined.

\subsection{Hydrate Formation with Fresh Water}

Induction time is calculated for the system of $89.4 \% \mathrm{C}_{1}+$ $10.6 \% \mathrm{C}_{2}$ and $1.5-7 \mathrm{~mm}$ fresh water droplets at $273.85 \mathrm{~K}$. Similar to Lee et al. experiments [6], the first three calculations are carried out at a formation pressure of 5.1 MPa and the last one is carried out at $3.6 \mathrm{MPa}$.

Temperature, pressure and gas composition reported by Lee et al. [6] are measured in the bulk of the gas and they are not the interfacial values. But, hydrate formation is initiated at the liquid gas interface which we have no information on. Moreover, uncertainty in the temperature or pressure measurement or unexpected interfacial phenomena may force the interface concentration be a different value in each experiment. Hence, the value of the induction time differs in one experiment compared to the other, because it is greatly influenced by the driving force which is a function of interface concentration.

To examine the above assumption, a sensitivity analysis is carried out to find the effect of driving force on the prediction of the induction time. It is shown that, $\pm 1.0 \%$ deviation of the driving force with respect to the value calculated by equation 15 at the bulk conditions can produce induction times which differ by 40-50 minutes. In the above calculations, driving force is changed in order to predict the experimental induction time reported by Lee $e t$ al. [6]. The predicted and experimental induction time are compared and shown in Table 3 . The results of Table 3 show that the induction times from the experiment can be matched by adjusting the driving force. Small changes in the driving force result in large changes in the induction times. Further, the results show that the induction time and the driving force of the droplets in the same experiment are nearly the same. It is concluded that in each experiment interface concentration playing the major rule and the droplet size has no noticeable effect on calculating the induction time.

Figures 3-8 show the calculated gas concentration profiles inside the water droplet and the water conversion to hydrate at the liquid-gas interface. Figures 3 and 4 show methane and ethane concentration profiles inside a $5 \mathrm{~mm}$ water droplet with respect to time and radial distance. It is shown that the interface concentration is different than the internal concentration of the droplet.

Figure 5 shows fraction of water conversion to hydrate for a $5 \mathrm{~mm}$ water droplet. It is shown that the time required for $1 \%$ conversion of water to hydrate is equal to 38 minutes. 
TABLE 3

Comparison of the calculated and experimental induction times at $273.85 \mathrm{~K}$ for the droplets without hydrate memory

\begin{tabular}{|c|c|c|c|c|c|}
\hline \multirow{2}{*}{ Water droplet } & \multicolumn{2}{|c|}{ Predicted (This work) } & \multicolumn{3}{|c|}{ Experimental (Lee et al. [6]) } \\
\hline & Ind. Time (min) & $\Delta g / k T$ & Ind. Time (min) & Experiment No. & Pressure (MPa) \\
\hline $7 \mathrm{~mm}$ & 79.0 & 0.997 & 79.3 & 4 & 3.6 \\
\hline \multirow[t]{2}{*}{$6 \mathrm{~mm}$} & 47.0 & 1.165 & 48.7 & 1 & \multirow{2}{*}{5.1} \\
\hline & 97.0 & 1.147 & 95.0 & 3 & \\
\hline \multirow[t]{2}{*}{$5 \mathrm{~mm}$} & 38.0 & 1.167 & 35.7 & 2 & 5.1 \\
\hline & 78.0 & 0.995 & 79.3 & 4 & 3.6 \\
\hline $2.5 \mathrm{~mm}$ & 47.0 & 1.156 & 47.2 & 1 & \multirow{4}{*}{5.1} \\
\hline & 93.0 & 1.140 & 94.3 & 3 & \\
\hline \multirow[t]{2}{*}{$1.5 \mathrm{~mm}$} & 47.0 & 1.151 & 47.8 & 1 & \\
\hline & 94.0 & 1.134 & 94.7 & 3 & \\
\hline
\end{tabular}

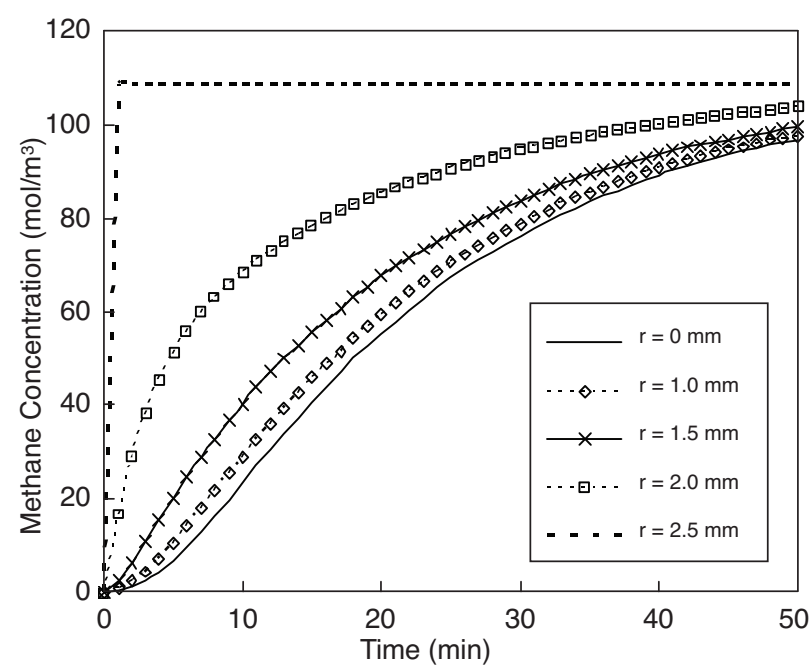

Figure 3

Methane concentration profile inside the water droplet for the gas mixture and a $5 \mathrm{~mm}$ water droplet at $273.85 \mathrm{~K}$ and 5.1 MPa.

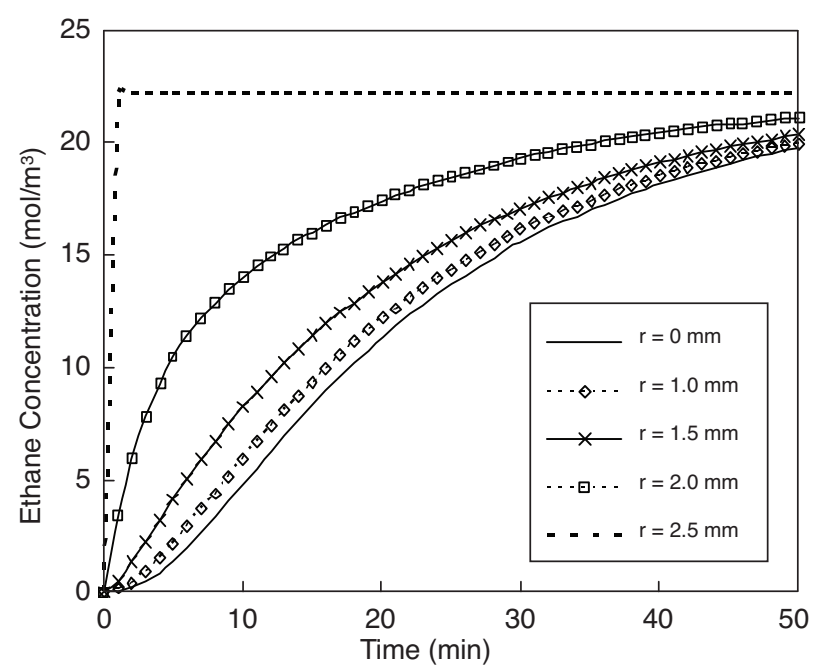

Figure 4

Ethane concentration profile inside the water droplet for the gas mixture and a $5 \mathrm{~mm}$ water droplet at $273.85 \mathrm{~K}$ and 5.1 MPa.
Figures 6 and 7 show methane and ethane concentration profiles inside a $6 \mathrm{~mm}$ water droplet with respect to time and radial distance. It is shown that the interface concentration is different than the internal concentration of the droplet.

Figure 8 shows fraction of water conversion to hydrate for a $6 \mathrm{~mm}$ water droplet. It is shown that the time required for $1 \%$ conversion of water to hydrate is equal to 47 minutes.

\subsection{Hydrate Formation with Hydrate Memory}

In Lee et al.'s [6] experiments, the droplets after first stage of hydrate formation are exposed to $1 \mathrm{Mpa}$ pressure for 1 hour at $273.85 \mathrm{~K}$ to decompose completely. Then, for the second stage of hydrate formation, they are exposed to 5.1 MPa for the first three experiments and to $3.6 \mathrm{MPa}$ for the fourth 
experiment at $273.85 \mathrm{~K}$. Due to the memory effect, the second induction times measured in four experiments are in the range of 0.5-1.8 minutes for all droplet size.

The memory effect is modeled based on the results of the work of Rodger [7] and Thompson et al. [8]. They did show that a low concentration of hydrate crystallite remains in the solution after complete melting of the hydrate [7, 8]. Hence, the remaining hydrate structures in the droplets play the rule of nucleation active centers for the second stage of hydrate formation. The remaining hydrate structures exposed their surface for the initiation of hydrate formation and the nucleus is formed on the solid substrate instead of liquid gas interface. The nucleus formed on solid substrate is cap shape instead of lens shape nucleus formed at liquid/gas interface. The difference between nucleus formation on solid substrate and at the liquid gas interface is shown in Figure 2.

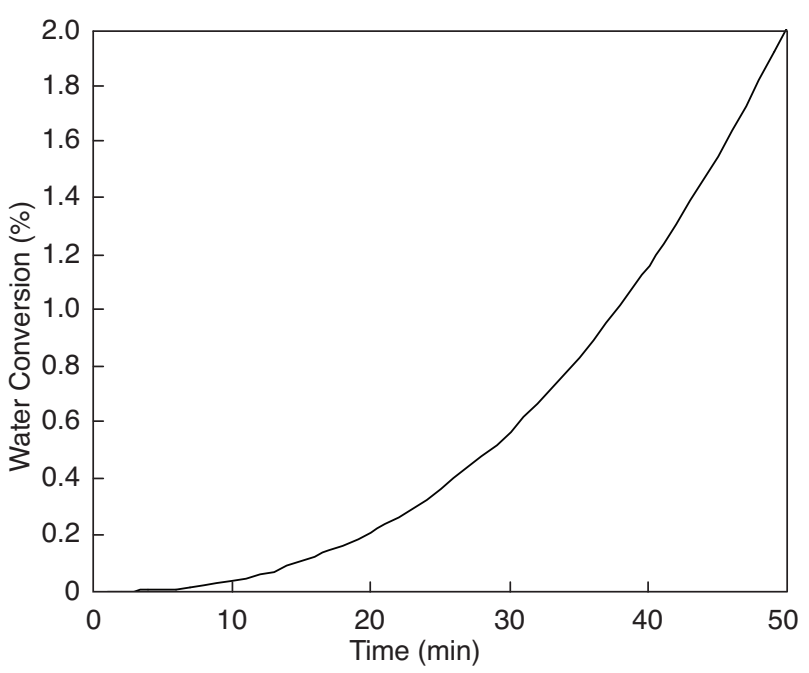

Figure 5

Fraction of water conversion to hydrate for the gas mixture and a $5 \mathrm{~mm}$ water droplet at $273.85 \mathrm{~K}$ and $5.1 \mathrm{MPa}$ $(\Delta g / k T=1.167)$.

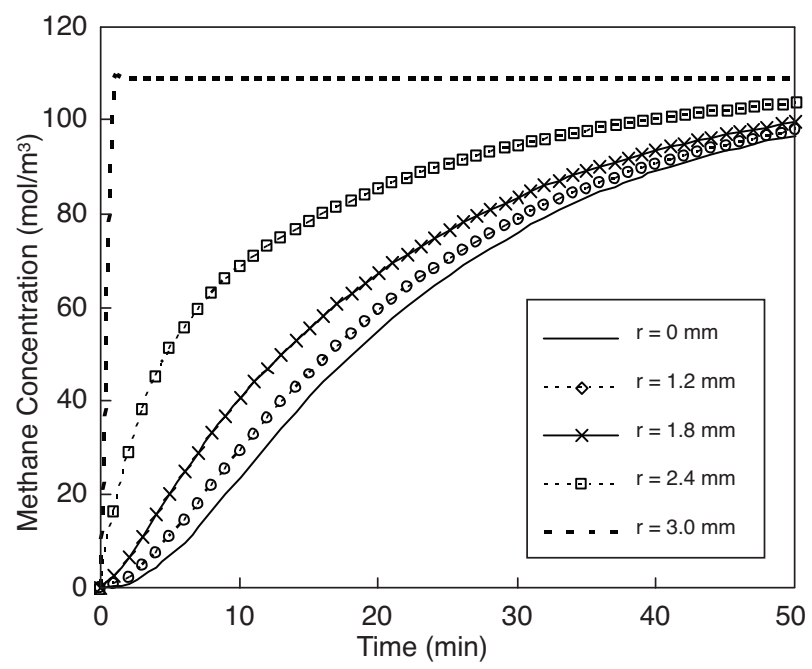

Figure 6

Methane concentration profile inside the water droplet for the gas mixture and a $6 \mathrm{~mm}$ water droplet at $273.85 \mathrm{~K}$ and 5.1 MPa.

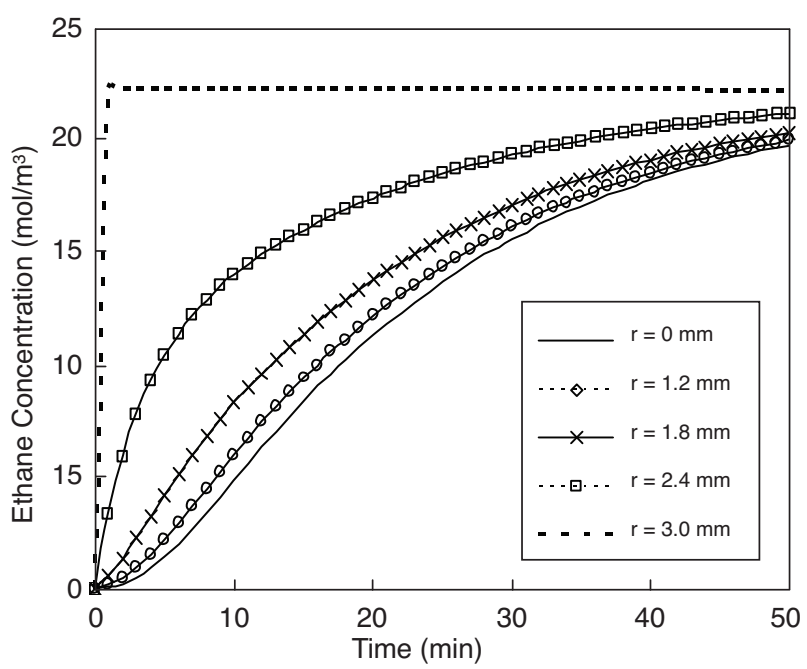

Figure 7

Ethane concentration profile inside the water droplet for the gas mixture and a $6 \mathrm{~mm}$ water droplet at $273.85 \mathrm{~K}$ and 5.1 MPa.

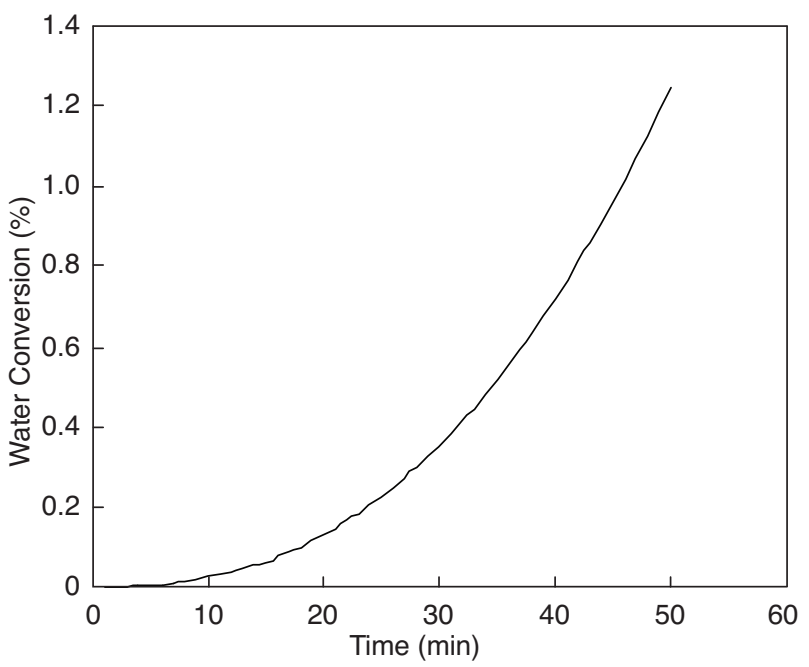

Figure 8

Fraction of water conversion to hydrate for the gas mixture and a $6 \mathrm{~mm}$ water droplet at $273.85 \mathrm{~K}$ and $5.1 \mathrm{MPa}$ $(\Delta g / k T=1.165)$. 
TABLE 4

Comparison of the calculated and experimental induction times at $273.85 \mathrm{~K}$ for the droplets with hydrate memory

\begin{tabular}{|c|c|c|c|c|c|}
\hline \multirow{2}{*}{ Water droplet } & \multicolumn{2}{|c|}{ Predicted (this work) } & \multicolumn{3}{|c|}{ Experimental (Lee et al. [6]) } \\
\hline & Ind. Time (min) & $C_{p} \times 10^{-10}\left(\mathrm{~m}^{-2}\right)$ & Ind. Time (min) & Experiment No. & Pressure (MPa) \\
\hline $7 \mathrm{~mm}$ & 2.20 & 2.83 & 2.3 & 4 & 3.6 \\
\hline \multirow[t]{2}{*}{$6 \mathrm{~mm}$} & 1.50 & 4.25 & 1.5 & 1 & \multirow{2}{*}{5.1} \\
\hline & 1.00 & 14.16 & 0.5 & 3 & \\
\hline \multirow[t]{2}{*}{$5 \mathrm{~mm}$} & 2.25 & 1.47 & 2.2 & 2 & 5.1 \\
\hline & 2.00 & 2.83 & 2.0 & 4 & 3.6 \\
\hline $2.5 \mathrm{~mm}$ & 1.67 & 1.42 & 1.7 & 1 & \multirow{4}{*}{5.1} \\
\hline & 1.05 & 5.66 & 1.0 & 3 & \\
\hline \multirow[t]{2}{*}{$1.5 \mathrm{~mm}$} & 1.75 & 0.78 & 1.8 & 1 & \\
\hline & 1.42 & 1.27 & 1.4 & 3 & \\
\hline
\end{tabular}

Nucleation rate is calculated again by Equation 7 with $A k$ parameter calculated by Equation 10 which is suitable for the nucleus formation from solution with nucleation active center. In Equation 10, $C_{p}$ is the concentration of the nucleation active centers and $A_{p}$ is their surface area. In order to determine the concentration of nucleation active center, $C_{p}$ is optimized to predict the experimental induction times. Constraint of this formulation is that $C_{p}$ values must not be less than the concentration of one hydrate unit cell per drop. $A_{p}$ is equal to the surface area of a hydrate unit cell which is equal to $1.7 \mathrm{~nm}^{2}$.

Induction times are calculated for a gas mixture of $89.4 \%$ $C_{1}+10.6 \% C_{2}$ and $1.5-7 \mathrm{~mm}$ water droplets with hydrate memory based on the optimized value of $C_{p}$ at $273.85 \mathrm{~K}$. Similar to Lee et al. experiments [6], the first three calculations are carried out at formation pressure of $5.1 \mathrm{MPa}$ and the last one is carried out at 3.6 MPa. The predicted and experimental induction times are compared in Table 4.

At the present time, there is not a solid reason to know why there is that much change in some cases in which we have to include factor of three in order to match the experimental data. But, this can be due to the fact that the number of the remaining hydrate structures even at the same thermodynamics conditions can be different from one experiment to another. Further, the water structure in the vicinity of decomposing hydrate is changing [8] which influences the number of the remaining hydrate structures in each experiment differently.

Although, this can be an explanation but the subject should be further considered in the future works.

\section{CONCLUSION}

Induction time for the hydrate formation of the gas mixture on a water droplet is calculated based on the model developed in this work. The calculated induction time for a mixture of
89.4\% methane $+10.6 \%$ ethane and $1.5-7 \mathrm{~mm}$ fresh water droplets at $273.85 \mathrm{~K}$ and $3.6-5.1 \mathrm{MPa}$ are greatly influenced by the driving force. It is found that the droplets with the same interfacial concentrations have similar driving force and similar induction times. The calculated induction times are independent of the droplet size as observed by Lee et al. [6].

The calculated induction time for the water droplet with hydrate memory are greatly influenced by the concentration of the remaining hydrate structures. The remaining structures change the nucleus formation mechanism and nucleation rate. The new mechanism of hydrate formation highly reduces the induction time.

\section{REFERENCES}

1 Sloan E.D. (1998) A Statistical Thermodynamic Approach to Hydrate Phase Equilibria: Clathrate Hydrates of Natural Gases, 2th edition, Marcel Dekker, New York.

2 Englezos P. (1993) Clathrate hydrates, Ind. Eng. Chem. Res. 32, 7, 1251-1274.

3 Kvamme B. (1996) A new theory for the kinetics of hydrate formation, 2th International conference on gas hydrates proceedings, Toulouse, France, June 2-6.

4 Kvamme B. (2000) A Unified Nucleation Theory for the Kinetics of Hydrate Formation, Ann. NY Acad. Sci. 912, 496-501.

5 Kashchiev D., Firoozabadi A. (2003) Induction time in crystallization of gas hydrates, J. Cryst. Growth 250, 499-515.

6 Lee J.D., Susilo R., Englezos P. (2005) Methane-ethane and methane-propane hydrate formation and decomposition on water droplets, Chem. Eng. Sci. 60, 4203-4212.

7 Rodger P.M. (2000) Methane- hydrate: Melting and memory, Ann. NY Acad. Sci.912, 474-782.

8 Thompson H., Alan K.S., Buchanan P., Aldiwan N., Creek J.L., Koh C.A. (2006) Methane hydrate formation and decomposition: Structural studies via neutron diffraction and empirical potential structure refinement, J. Chem. Phys. 124, 164507-1-7. 
9 Kashchiev D. (2000) Overall Crystallization: Nucleation, Basic Theory with Applications, Butterworth-Heinemann, Oxford.

10 Volmer M. (1939) Kinetic Analysis of Typical Special Cases with Experimental Verification: Kinetics of Phase Formation (Kinetik der Phasenbildung), Steinkopff, Dresden.

11 Mullin J.W. (1997) Crystal growth: Crystallization, Butterworth-Heinemann, Oxford.

12 Kashchiev D., Firoozabadi A. (2002) Nucleation of gas hydrates, J. Cryst. Growth 243, 476-489.

13 Anklam M.R., Firoozabadi A. (2004) Driving force and composition for multicomponent gas hydrate nucleation from supersaturated aqueous solutions, J. Chem. Phys. 121, 23, 11867-11875.
14 Van der Waals J.H., Platteeuw J.C. (1959) Clathrate Solutions, Adv. Chem. Phys. 2, 1, 1-57.

15 Parrish W.R., Prausnitz J.M. (1972) Dissociation pressures of gas hydrates formed by gas mixtures, Ind. Eng. Chem. Process Design Development 11, 26, 26-35.

16 Soave G. (1972) Equilibrium constants from a modified RedlichKwong equation of state, Chem. Eng. Sci.27, 6, 1197-1203.

17 Bird R.C., Prausnitz J.M., Poling B.E. (1987) Diffusion Coefficients: The properties of gases and liquids, 4th edition, Mc Graw Hill book company, New York.

Final manuscript received in January 2008 Published online in September 2008 